\title{
Molecular Docking Analysis of Phytochemical Thymoquinone as a Therapeutic Agent on SARS-Cov-2 Envelope Protein
}

\author{
Abdul Khader Sultan Mohideen 1,* (D) \\ 1 Faculty of Zoology, The New College (Autonomous), Chennai-600014, Affiliated to University of Madras, Tamil Nadu, \\ India \\ * Correspondence: smdbio@yahoo.co.in;
}

Scopus Author ID 55260117700

Received: 9.06.2020; Revised: 18.07.2020; Accepted: 20.07.2020; Published: 23.07.2020

\begin{abstract}
The sudden outbreak due to severe acute respiratory syndrome coronavirus 2 (SARS- Cov2) is responsible for causing acute, highly dreadful coronavirus disease (COVID-19). The pore-forming proteins in the SARS- CoV-2 envelope protein employ amphipathic $\alpha$-helix for pore formation. The pore openings are essential for the transport of ions, toxins, and viroporin activity. Moreover, there is an insurgence to identify lead compounds to target the novel coronavirus for therapeutic purposes. Therefore in the present study, the SARS CoV-2 envelope protein sequence was analyzed, constructed the three-dimensional homology model, and screened against the bioactive phytochemical Thymoquinone (TQ). Molecular docking was performed between the modeled E protein and TQ to study protein-ligand interactions using ArgusLab 4.0. The investigation revealed that the modeled E protein contains a single $\alpha$-amphipathic helix identified for the first time across the Amino-terminal region of the transmembrane domain may contribute to pore formation of small membrane proteins. Molecular docking results showed the promising inhibitory potential of the ligand TQ and the binding free energy of the bound complex was found to be $-9.01 \mathrm{kcal} / \mathrm{mol}$. The in silico approach has explicitly demonstrated the significant inhibitory effects of the ligand TQ. Therefore it may be used effectively as an antagonist against the SARS- CoV-2 infection owing to its outstanding pharmacological properties.
\end{abstract}

Keywords: Coronavirus; SARS-CoV-2 Envelope protein; Phytoconstituent; Thymoquinone; Bioactive compound; Homology modeling; Molecular docking.

(C) 2020 by the authors. This article is an open-access article distributed under the terms and conditions of the Creative Commons Attribution (CC BY) license (https://creativecommons.org/licenses/by/4.0/).

\section{Introduction}

The sudden outbreak of novel coronavirus $(\mathrm{CoV})$ has completely appalled across the world due to its pandemic nature. The coronavirus, also called severe acute respiratory syndrome coronavirus 2 (SARS-CoV-2) appeared in the month of December 2019 at Wuhan, China. This virus is highly pathogenic, which is responsible for causing serious, lifethreatening pneumonia coronavirus disease 2019 (COVID-19). Infected patients show symptoms of respiratory discomfort and dyspnoea. However, these viruses are not only confined to the respiratory tract but may also affect the central nervous system [1].

$\mathrm{CoVs}$ are enveloped viruses consisting of a single-stranded RNA as its genome. The size of the genome ranges from 26 to 32 kilobases, regarded as one of the largest among retroviruses [2]. The most striking feature of coronavirus is the presence of club-shaped spikes 
that protrude from its surface [3]. CoVs infect a wide range of avian and mammalian species and can cause severe illness in humans [4].

Therefore, there is an insurgence to identify natural chemical compounds possessing pharmacological properties expressing a high degree of safety, stability, and efficacy. Medicinal plants have always been the natural source of different phytoconstituents, inevitably used as contemporary drugs that have found their presence in modern therapeutic approaches [5]. They are used to heal and cure human diseases due to the presence of phytochemical constituents [6].

Phytoconstituents occur naturally in various parts of medicinal plants like leaves, flowers, vegetables, bark, and roots that show defense mechanisms against various diseases [7]. The important phytoconstituents of plants include tannins, resins, lectins, alkaloids, flavonoids, phenolic compounds glycosides, etoposides, tanniposides, fatty acids, waxes, terpenoids, phenolic acids, and polypropanoids, etc., [8,9]. These constituents also contain several moieties like naphthoquinone, anthraquinones, and benzoquinone, which facilitate biological or pharmacological interaction with its substrate, essential for drug design and development [10].

In the present study, Nigella sativa L. (N. sativa) was selected due to its outstanding pharmacological and biological activities. $N$. sativa is also known as black seed or kalonji, is an annual flowering plant in the family Ranunculaceae. It is native to the Indian Subcontinent and West Asia widely used as a medicinal plant throughout the world [11]. It is popularly adopted in traditional systems of medicine such as Unani, Ayurveda, and Siddha. The seeds and its oil have an age-old history of folklore applied in different systems of food and medicine [12]. In Islamic literature, it is recognized as one of the indispensable forms of holistic medicine. It has been recommended to use on a regular basis in Tibb-e-Nabwi, Prophetic Medicine [13]. In the Middle East, the seeds of $N$. sativa are used for dietary purposes. Moreover, the biological activities of $N$. sativa seeds are mainly due to the presence of essential oil constituent thymoquinone (TQ) ranging from 30-48\%, which was first obtained by ElDakhakhny [14].

TQ is a major bioactive compound present in black seed oil. The black seed oil is listed in the United States Food and Drug Administration (USFDA) and is "Generally Recognized as Safe."[15] and has received tremendous significance for its pharmacological properties and therapeutic potential [16]. The major pharmacological activities expressed by TQ are antimicrobial, anti-cancer, anti-inflammatory, antioxidant, etc. [17]. Furthermore, the topical application of $N$. sativa oil was found to be effective with minimal side effects during the treatment and combat of allergic rhinitis [18]. Moreover, the miraculous power of healing has fetched $N$. sativa, a place among the top-ranked evidence-based herbal medicines. This is mostly attributed due to the presence of active ingredients of the essential oil TQ, which has therapeutic effects [12].

Many researchers have carried out extensive work in unraveling SARS CoV structural proteins. The structural proteins like envelope $(\mathrm{E})$ protein, spike $(\mathrm{S})$ protein, and membrane (M) protein can be used as suitable candidates to study drug-target interactions. The SARS$\mathrm{CoV}$ E protein is made of 76 amino acids consisting of one alpha-helical transmembrane (TM) region. The TM domain is made of 25 amino acid residues [19]. The E proteins are integrated with the lipidic virion layer, along with the $S$ and $M$ proteins [20].

A recent study pertaining to the screening of medicinal plant compounds showed 32,297 potential antiviral phytochemicals, which may inhibit the SARS-CoV-2 3CLpro 
enzyme, thereby facilitating rapid drug discovery against COVID-19 pandemic [21]. In the same manner, inhibition of the ion channel activity in SARS CoV E protein may be used as a suitable target for developing new antiviral drugs [22]. In fact, experimental studies also suggest that defects in the SARS CoV E gene in a mouse model expressing SARS-CoV human ACE2 (angiotensin-converting enzyme-2) receptor failed to grow in the cerebrospinal nervous system [23]. Similarly, the lack of E protein led to the obstruction of virus interaction in the secretory pathway and inhibition of virus maturation [24, 25].

Thus, the main objective of this study is to search for novel bioactive compounds with therapeutic properties. Though crystal structures of the E protein from different organisms have been determined earlier, there is still a need for structural information. Hence, the present investigation is focused on the homology modeling of SARS-CoV-2 E protein to gain insights on its structural and functional aspects. Molecular docking is performed between SARS-CoV$2 \mathrm{E}$ protein and the phytoconstituent TQ to study the protein-ligand interactions by adopting in silico strategies to elucidate its antiviral properties for medicinal purposes.

\section{Materials and Methods}

\subsection{Sequence retrieval and analyses.}

The amino acid sequence of SARS-Cov-2 envelope protein was extracted from GenBank: QHD43418.1 [26] and stored in FASTA format for further analysis (Fig.1).

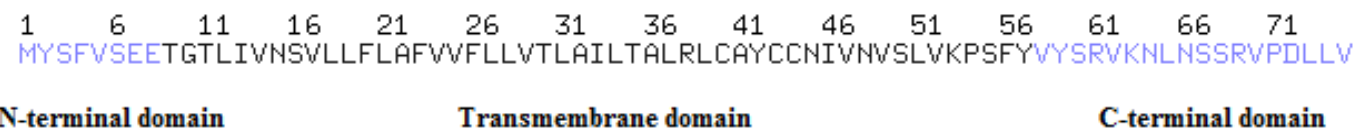

Figure 1. The amino acid sequence of SARS-CoV-2 E protein (residues 1-75) extracted from GenBank (QHD43418.1) showing different domains.

Physico-chemical properties of the SARS-Cov-2 envelope protein were computed by the ProtParam tool (http://web.expasy.org/protparam/), to analyze the molecular weight, theoretical pI, amino acid composition, and grand average of hydropathicity (GRAVY). The antigenicity of the E protein was predicted with AllerTOP v.2.0 [27]. SOPMA server was used to predict the secondary structure of E protein to evaluate the conformational information about the location of the $\alpha$-helices, $\beta$-strands, turns, loops, random, and coils from the protein structure [28].

\subsection{Homology modeling of SARS-Cov-2 E protein.}

BLASTp search of SARS-Cov-2 E protein using the default parameter was conducted against the Brookhaven Protein Data Bank (PDB) to find a suitable template for comparative or homology modeling (http://blast.ncbi.nlm.nih.gov/). The PDB structures were selected based on sequence coverage and identity, and stored in PDB format for further analysis. The three dimensional (3D) structure of SARS-Cov-2 E protein was predicted using the GalaxyTBM server [29]. The software generated several models through an optimization-based refinement method. The model with the lowest energy was selected for further analysis. The predicted structure was visualized using PyMOL molecular graphics viewer v.2.2.0 (www.pymol.org). Further, the structural domain regions were predicted with the help of the PSIPRED server (http://bioinf.cs.ucl.ac.uk/psipred/). 


\subsection{Structural validation of the modeled SARS-Cov-2 E protein.}

The predicted 3D structure of the model was determined with the help of PROCHECK, Structural analysis, and verification server (SAVES) (http://nihserver.mbi.ucla.edu/SAVES) which estimates the stereochemical aspects besides the main chain and side chain parameters with detailed analysis [30].

The quality of the model was assessed for various parameters, which include Root Mean Square Deviation (RMSD) using TM-score (https://zhanglab.ccmb.med.umich.edu/TMscore/), Errat for estimating the progress of crystallographic model building and refinement, ProSA for the prediction of Z-score value using a suitable template [31].

\subsection{Functional assessment of SARS-Cov-2 E protein.}

The functional assessment of the modeled E protein was performed using the DIAL server [32], and the active site amino acid residues were predicted with the help of the Galaxy server [29].

\subsection{Ligand preparation and molecular docking.}

The molecular structure of the ligand TQ was extracted in SDL format from the Brookhaven protein data bank using the PDB ID: 4HCO [33]. The ligand was removed from the complex and then converted into a standard PDB format by applying OpenBabel v.2.3.2 (http://openbabel.org) (Fig. 2) for docking the catalytic site of the modeled SARS- Cov-2 E protein by applying ArgusLab software v4.0 [34]. In the process of docking, the ligand-binding sites of the modeled E protein were prepared for TQ based on the Galaxy server results obtained before molecular docking. The ArgusLab program has a built-in empirical scoring function (AScore) and docking engine (ArgusDock). The energy minimization was carried out using the universal force field (UFF) molecular mechanics. The calculations were adjusted to dock mode and flexible model with a grid resolution of $0.40 \AA$. The pose with the lowest binding energy was considered for calculating the scoring function, which shows the modeled E protein-ligand interactions. The different binding modes between the bound complexes were observed through PyMOL V.2.3.3 (www.pymol.org). The docking was further confirmed by uploading TQ and the modeled E protein to the PatchDock server [35].

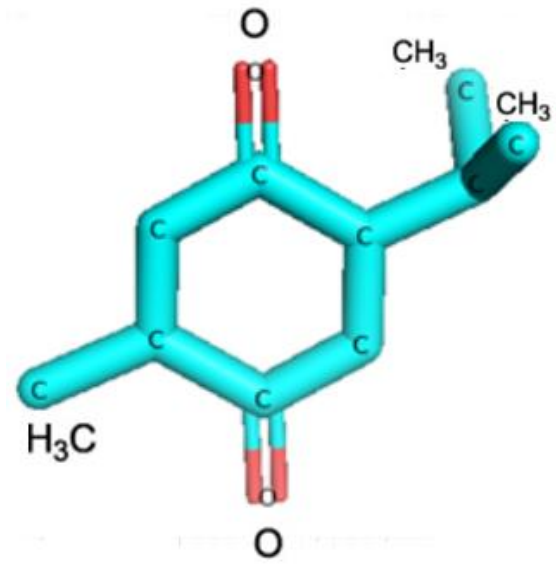

Figure 2. Molecular structure of the phytoconstituent Thymoquinone (TQ) obtained from PDB ID: 4HCO is used effectively as an inhibitor owing to its biological and pharmacological activities. 


\section{Results and Discussion}

\subsection{Physico-chemical properties and amino acid composition of SARS-Cov-2 E protein.}

The SARS- Cov-2 E protein has a molecular weight of $83.6 \mathrm{kDa}$, and the theoretical isoelectric point (pI) was found to be 8.57. Some substances like viruses and certain synthetic polymers have very high molecular weights. In fact, the molecular weight of protein plays a vital role in the prediction of protein structure [36]. The theoretical pI exhibits its alkaline nature ( $\mathrm{pI}>7$ ) due to the presence of basic amino acids, lysine, and arginine. These basic amino acids are involved in membrane protein activity and recognition of membrane voltages [37]. The GRAVY value was found to be 1.128 , which is $\geq 1$, indicating its hydrophilic nature and affinity towards water molecules [38]. The modeled $\mathrm{E}$ protein identified as a non-allergen and resembles with $\mathrm{C}-\mathrm{C}$ chemokine receptor type $1, \mathrm{C}-\mathrm{C}$ chemokines do not have an amino acid between the first two cysteines of the characteristic four-cysteine motif [39]. Furthermore, it binds to the human macrophage inflammatory protein 1 alpha/RANTES receptor [40]. Subsequently, it transduces a signal by increasing the intracellular calcium ions level, responsible for affecting stem cell proliferation.

The secondary structure predicted by GOR4 (with default parameters) from the SARSCov-2 E protein demonstrated that the modeled E protein having the composition of Helix = $33.33 \%$, Coil $=53.33 \%$, Strand $=13.33 \%$ and Beta-turn $=0.00 \%$ respectively. It is quite obvious from this secondary structure prediction that the modeled E protein belongs to the $\alpha$ helical class of proteins.

\subsection{Modeled structure of SARS-Cov-2 E protein.}

The 3D structure of SARS-Cov-2 E protein was predicted by homology modeling. BLASTp analysis (http://www.ncbi.nlm.nih.gov/) of the amino acid sequence of the modeled E protein against the PDB database revealed good alignment with the crystal structure of microbial origin, PDB ID: 5X29_A [41]. The best template PDB ID: 5X29_A was assessed based on query coverage (82)\%, an E-value (1e-30), and sequence identity (89\%). The model with the least energy $(-6.61)$ predicted by the GalaxyTBM server was considered for further analysis. The 3D structure of the modeled E protein mainly composed of $\alpha$-helices, loops, or $\beta$ turns (Fig.3). Interestingly, the predicted 3D structure of SARS-Cov-2 E protein has the characteristic features of the coronaviridae family, designated as an integral small membrane protein.

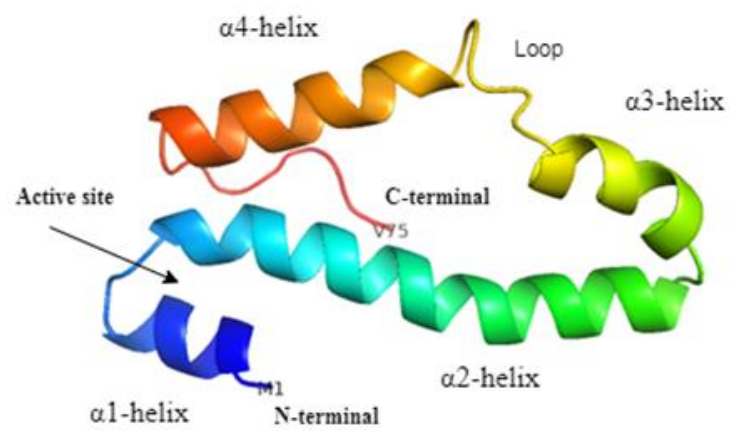

Figure 3. Three-dimensional structure of SARS- Cov-2 E protein is made of four $\alpha$-helices, loops, or $\beta$ turns. The active sites of the residues are present between $\alpha 1$ and $\alpha 2$-helices. The $\mathrm{N}$ and $\mathrm{C}$-terminal shown in blue and red color occupy at the extracellular region. 


\subsection{Description of SARS-Cov-2 E protein structure.}

The modeled SARS-Cov-2 E proteins are a short integral membrane protein, which contains three domains, namely N-terminal domain, transmembrane domain (TMD), and Cterminal domain (residues 1-75). The modeled E protein is consisting mainly of four righthanded $\alpha$-helices connected in between loops or $\beta$-turns. The $\alpha$-helices are the simplest and most common form of protein structural motifs that occurs in a helix bundle. This type of arrangement helps in DNA binding motifs, including helix-turn-helix motifs and zinc finger motifs [42].

The N-terminal domain with $\alpha-1$ (residues 1-8) situated on one side that forms the extracellular space known as accessible molecular surface or solvent-exposed area. The TMD consists of 2 subunits, namely S1 and S2 made of $\alpha-2$ (residues 9-38) and $\alpha-3$ (residues 42-57) helices, aligned parallel and connected by a short loop or $\beta$ turn (residues 39-41). The loop residues are polar and hydrophobic, lies within the cytoplasmic region-the $\alpha-3$ shares partly by a transmembrane helix and pore-lining helix of the TMD. The presence of a short loop or $\beta$ turn is a characteristic feature of thermophilic organisms that provides the extra protein stability at high temperatures, thereby preventing unfolding and denaturation [43]. The N-terminus of the TMD contains a single $\alpha$-amphipathic helix made of alternative hydrophobic/hydrophilic residues, identified as a structural motif. The structural motif has the amino acid sequence VSEETG (residue number 5-10) where $\mathrm{V}$ and $\mathrm{G}$ are hydrophobic and in between the polar residues are arranged. While, in TLIVN (residue number 11-15), TN are hydrophilic and in between non-polar residues are present. The $\alpha-4$ helix (residues 58-75) is positioned extracellular on the C-terminal domain of the SARS- Cov-2 E protein (Fig. 4).

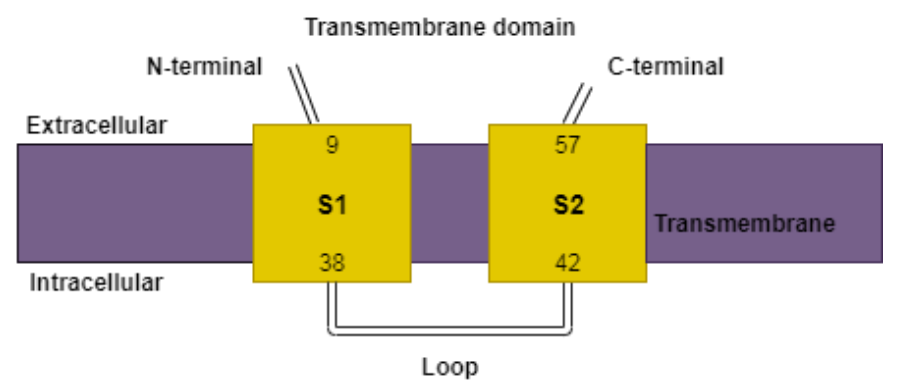

Figure 4. The SARS-CoV-2 E protein is comprising of three domains: the N-terminal domain, the transmembrane domain (TMD), and the C-terminal domain. The TMD consists of two subunits, namely S1 (residues 9-38) and S2 (residues 42-57) connected by a loop.

Moreover, the predicted single amphipathic $\alpha$-helix at the N-terminus of TMD is located very close to the surface of the lipid membrane, essential for protein-ligand interactions. Moreover, the secondary structural elements ( $\alpha$-helices) may be involved in transmembrane pore formation or pore-forming proteins (PFPs), which employs amphipathic $\alpha$-helix for pore formation. The pore openings allow the movement of ions and other components like toxins via the cell membrane barrier [44]. This is in agreement with the pore-forming domain consisting of hydrophobic helical hairpin buried between two amphipathic layers of $\alpha$-helices, as observed in Bax and colicins [45]. Similarly, it is also seen in the CoV E protein TMD consisting of one amphipathic $\alpha$-helix that oligomerizes to form an ion-conductive pore in membranes [46]. Moreover, they can affect the host cell's physiology, tissue integrity, and inflammatory responses that may inhibit antimicrobial treatment $[47,48]$. 
Furthermore, computational approaches and spectroscopic studies demonstrate that the SARS-CoV E TMD goes through oligomerization, which is a characteristic feature of ionchanneling proteins [49]. In addition, the synthetic peptides of SARS-CoV E viroporin are capable of transporting $\mathrm{Ca} 2+$ associated with the inflammatory response mostly seen in acute respiratory distress syndrome [50]. A similar mechanism was also observed with other viral proteins that might affect or modulate the oligomerization of Infectious bronchitis virus $\mathrm{E}$ protein. Furthermore, the M2 protein amphipathic helix motif is necessary for the release of Influenza A virus particles needed for the formation of budding, scission, and viroporin activity [51].

\subsection{Protein model validity.}

The geometrical and structural consistency of the modeled SARS-Cov-2 E protein was evaluated at the SAVES server. The phi and psi angle distributions of the Ramachandran map using PROCHECK revealed that $92.9 \%$ and $5.7 \%$ amino acid residues are located in the most favored and additionally allowed regions, while $1.4 \%$ and $0.0 \%$ amino acid residues in the generously allowed and disallowed regions. This represents the geometric fitness of the modeled E protein (Fig. 5).

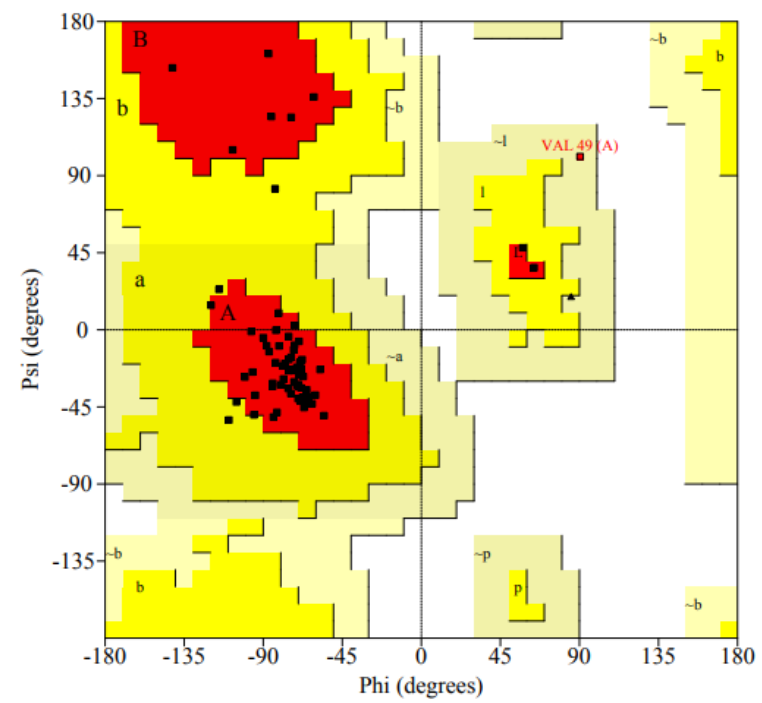

Figure 5. The Ramachandran map for SARS-Cov-2 E protein displays the phi-psi torsion angles of all the amino acid residues. The color shading on the map shows different regions. The red color conforms to the core regions exhibiting the most favorable combinations of phi-psi values.

This is in conformity with the SARS Coronavirus E protein pentameric ion channel, which exhibited $83.3 \%$ and $15.6 \%$ residues in the most favored and additionally allowed regions, while $1.1 \%$ and $0.0 \%$ residues were found in the generously allowed and disallowed regions (PDB: 5X29_A). Furthermore, PROCHECK analysis showed both the main and side chain parameters are within the acceptable range suggesting the reliability of the SARS-Cov-2 E protein structure.

Further, the calculation of overall RMSD and TM score between the modeled SARSCov-2 E protein and 5X29_A was found to be $1.24 \AA$ and 0.7 , respectively. It confirmed a pair of best-aligned chains between the template and predicted model, indicative of a perfect match between two structures. ERRAT map showed an overall quality factor of $74.24 \%$, which is indicative of a good resolution structure. The $\mathrm{Z}$ score was found to be -0.35 in SARS-Cov-2 E protein and 0.59 in PDB ID: 5X29_A (Fig. 6 ). 

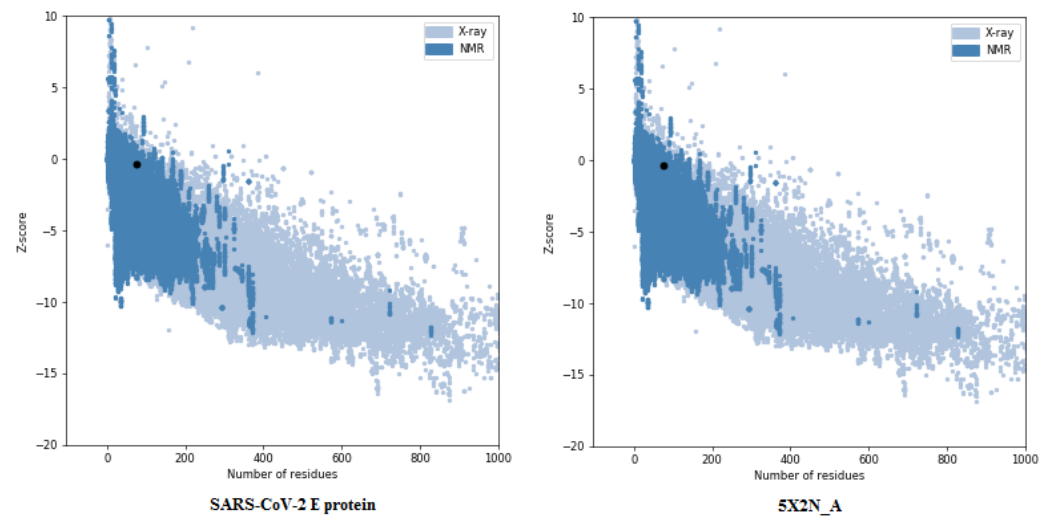

Figure 6. ProSA-web z-scores of the SARS-CoV-2 E protein and the template 5X2N_A in PDB calculated by X-ray crystallography (light blue) or NMR spectroscopy (dark blue) corresponding to their length. The plot exhibits only chains $<1000$ residues and a z-score $\leq 10$. The z-scores of modeled E protein and template are highlighted as large dots.

The predicted 3D model of SARS- Cov-2 E protein after validation was deposited in the Protein Model Database (PMDB). The modeled E protein can be retrieved using the Accession No. PM0082981 (https://bioinformatics.cineca.it/PMDB).

\subsection{Functional characterization of the predicted structure.}

The active site residues of the modeled SARS-Cov-2 E protein were found to be S6, $\mathrm{T} 9$, T11, I13, and V17. The monomeric form of modeled $\mathrm{E}$ protein contains three functional motifs, which include two N-glycosylation sites -located from the residues 48-51(NVSL), 6669 (NSSR) and one Protein kinase C phosphorylation site from the residues 67-69 (SSR) respectively. The modeled $\mathrm{E}$ protein also represented two $\mathrm{N}$-glycosylation sites along the subunit S2 and C-terminal region where carbohydrates may be linked to proteins with the help of asparagine ( $\mathrm{N}$-linked). In fact, asparagine residue can derive an oligosaccharide only if the residue is an integral part of L-Asn-X-Ser or L-Asn-X-Thr amino acid sequence where ' $\mathrm{X}$ ' can be any amino acid residue except proline. Glycosylation is responsible for maintaining protein stability in the blood. On the contrary, unglycosylated protein contains only $10 \%$ of the biological activity of the glycosylated form, and it gets easily removed from the system by the kidneys. Apart from that, potential glycosylation sites play an important role in adding sugars, enabling the protein to be more hydrophilic to take part in interactions with other proteins [52].

\subsection{Docking of SARS-Cov-2 E protein to ligand TQ.}

When docking runs were executed, the SARS-Cov-2 E protein showed tremendous binding affinity and a remarkable preference to its ligand TQ from the N-terminal domain (Fig 7). The binding energy was found to be $-9.01 \mathrm{kcal} / \mathrm{mol}$ using Argus Lab 4.0. The findings were further confirmed by the PatchDock server showing similar binding features. The estimated global energy was found to be $-32.03 \mathrm{kcal} / \mathrm{mol}$. In fact, negative free binding energy value confirms the modeled E protein and TQ complex to be more stable.

The structure-activity relationship is well illustrated in the present study between the SARS-Cov-2 E protein and ligand TQ interactions. In fact, a number of distinctive characteristics are linked with the active residues of the modeled E protein. TQ access to the binding residues is aided through the $\mathrm{N}$ - terminal domain through $\alpha 1$ and $\alpha 2$ helices of the modeled E protein. 
The surrounding residues of SARS-Cov-2 E protein and TQ are displayed within $8 \AA$ radius sphere. The modeled E protein residues, S6, T9, T11, I13, and V17 are close to its ligand $\mathrm{TQ}$ (central residue) along the $\mathrm{N}$ and $\mathrm{C}$ direction contribute to short-range interactions with the bound TQ (Fig. 7).

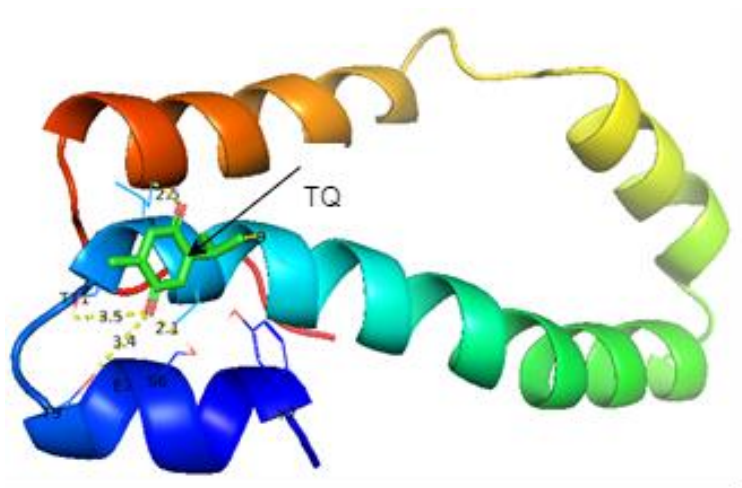

Figure 7. Docked complex of SARS- Cov-2 E protein and phytoconstituent Thymoquinone (TQ) obtained from Argus Lab v.4.0 demonstrating protein-ligand interactions. The central residue TQ is in close contact with the active site residues of the modeled E protein.

In the protein-ligand interactions, the side chains of the SARS-CoV-2 modeled E protein residues S6, T9, T11, I13, and V17 showed a close affinity to the bound ligand TQ. The distance between the hydrogen bond lengths was measured between the modeled E protein and ligand TQ (Fig. 8). It was observed from the docked complex that the $\mathrm{O}$ atom of the ligand TQ could act as a nucleophile on the side chain residues of modeled E protein. Therefore, the $\mathrm{O}$ atom of TQ when it comes in contact with the HG atom of S6 may trigger a chemical reaction leading to the reduction of oxygen and oxidation of hydrogen molecules. This may result in the formation of water or hydrogen peroxide as end products [53].

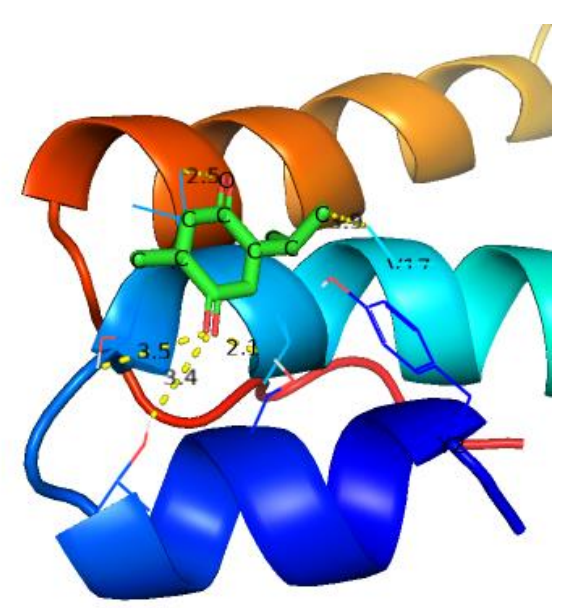

Figure 8. Molecular interactions between SARS-Cov-2 E protein and ligand TQ. The distance between the hydrogen bond lengths was calculated between the modeled E protein and ligand TQ The O atom (oxygen) of the TQ (Central residue) is in close association with the side chain HG atom (Gamma hydrogen) of the residue S6, may act as a potential nucleophile.

In addition, the HG1 and HG2 atoms of protein residues T9 and T11 base pairs with $\mathrm{O}$ atom of ligand TQ, which are polar and capable of forming $\mathrm{H}$ bonds as proton donors or acceptors, normally found in close proximity to the surface of protein or lipid membranes. In contrast, the CD1 atom of the protein residue I13 interacts with the $\mathrm{O}$ atom of ligand TQ and establishes Carbon-Oxygen hydrogen bonding ( $\mathrm{CH}-\mathrm{O})$ between them. Klaholz and Moras 
demonstrated protein-ligand binding mechanism in nuclear retinoic acid receptor bound to retinoid SR11254 to identify $\mathrm{CH}-\mathrm{O}$ hydrogen bonds in ligand recognition. Subsequently, they discovered several $\mathrm{CH}-\mathrm{O}$ bonds among different moieties of the ligand with hydrogen bond donors/acceptors at different positions, showing multiple $\mathrm{CH}-\mathrm{O}$ interactions may significantly facilitate ligand binding affinity [54]. Thus, it is clearly demonstrated that $\mathrm{CH}-\mathrm{O}$ bonding significantly contributes to the ligand-protein binding. Furthermore, the $\mathrm{C}$ atom of the protein residue V17 interacts with the $\mathrm{C}$ atom of ligand TQ. It should be noted that $\mathrm{C}-\mathrm{C}$ interactions are considered to be weak. However, polarization provides stability to the bound complex [55]. Based on these correlations, it was identified that the modeled $\mathrm{E}$ protein residues have a definite role in protein-ligand interactions (Table 1).

Table 1. Molecular Interactions between the ligand Thymoquinone (TQ) and SARS- Cov-2 E protein.

\begin{tabular}{l|c|c|c|c} 
S.No. & Ligand & Residue and atom & Ligand atom & Bond length $\left(\mathbf{A}^{\circ}\right)$ \\
\hline 1 & TQ & S6 \& HG & O & 2.1 \\
\hline 2 & & T9 \& HG1 & O & 3.4 \\
\hline 3 & & T11 \& HG1 & O & 3.5 \\
& & CG2 & C & 3.0 \\
\hline 4 & & I13 \& CD1 & O & 2.4 \\
\hline 5 & & V 17 \& CG2 & C & 3.9
\end{tabular}

Thus it is explicitly demonstrated from the docked complex that the modeled E protein displayed tremendous affinity to its ligand TQ and can be used effectively as a potential inhibitor against SARS-CoV-2 E protein. Moreover, TQ can enhance the synthesis of reactive oxygen species (ROS) in cells [56] that can be deleterious to DNA, RNA, and proteins, which may eventually cause cell death [57]. Above all, TQ has been clinically tested for its pharmacological activities against various types of ailments, including human carcinomas, as it shows negligible toxicity against normal cells [58]. Therefore, the search for novel antiviral agents to block COVID-19 is an immediate requirement, as there is no approved medicine for COVID-19 to date [59].

\section{Conclusions}

To sum up, the modeled 3D structure of the SARS-CoV-2 E protein provides comprehensive structural and functional details. More importantly, the structural motif identified for the first time across the N-terminal region of the TMD contains a single $\alpha$ amphipathic helix that may play a significant role in pore formation of small membrane proteins essential for the transport mechanism of ions, toxic substances, and viroporin activity. The docked complex between the SARS-CoV-2 E protein and ligand TQ displayed tremendous interactions suggesting potential inhibitory effects of the phytoconstituent. Thus, this in silico based approach has explicitly demonstrated the bioactive phytochemicals may inhibit the SARS-CoV-2 E protein ion channel, pore-forming protein activity. The present investigation may be used as a baseline to further develop TQ into a potential antiviral drug against the COVID-19 through suitable in-vitro and in-vivo models for drug transport through oral and intranasal medications.

\section{Funding}

This research received no external funding. 


\section{Acknowledgments}

The author would like to thank the Management, Principal, and Head, PG \& Research Department of Zoology, The New College (Autonomous), Chennai-600014, Affiliated to the University of Madras, India for their support and encouragement.

\section{Conflicts of Interest}

The authors declare no conflict of interest.

\section{References}

1. Li, Y.C.; Bai, W.Z.; Hashikawa, T. The neuroinvasive potential of SARS-CoV2 may play a role in the respiratory failure of COVID-19 patients. Journal of Medical Virology 2020, 92, 552-555, https://doi.org/10.1002/jmv.25728.

2. Woo, P.C.Y.; Huang, Y.; Lau, S.K.P.; Yuen, K.Y. Coronavirus Genomics and Bioinformatics Analysis. Viruses 2010, 2, 1804-1820,https://doi.org/10.3390/v2081803.

3. Almeida, J.D.; Berry, D.M; Cunningham, C.H.; Hamre, D.; Hofstad, M.S.; Mallucci, L.; McIntosh, K.; Tyrrell, D.A. Virology: Coronaviruses. Nature 1968, 220, 650-650, https://doi.org/10.1038/220650b0.

4. Ruch, T.R.; Machamer, C.E. The Coronavirus E Protein: Assembly and Beyond. Viruses 2012, 4, 363-382. https://doi.org/10.3390/v4030363.

5. Kaur, G.; Kataria, H.; Mishra, R. Medicinal Plants as Novel Promising Therapeutics for Neuroprotection and Neuroregeneration. In: New Age Herbals: Resource; Quality and Pharmacognosy. Singh, B.; Peter, K.V. eds. Singapore: Springer 2018; pp. 437-453.

6. Nostro, A.; Germanò, M.P.; D’Angelo, V.; Marino, A.; Cannatelli, M.A. Extraction methods and bioautography for evaluation of medicinal plant antimicrobial activity. Letters in Applied Microbiology 2000, 30, 379-384, https://doi.org/10.1046/j.1472-765x.2000.00731.x.

7. Krishnaiah, D.; Sarbatly, R.; Bono, A. Phytochemical antioxidants for health and medicine - A move towards nature. Biotechnol Mol Biol Rev 2007, 1, 97-104.

8. Stefania, S.; Filippo, M.; Marcello, N.; Valeria, B.; Stefano, D.A. New Drugs from Old Natural Compounds: Scarcely Investigated Sesquiterpenes as New Possible Therapeutic Agents. Current Medicinal Chemistry 2018, 25, 1241-1258, https://doi.org/10.2174/0929867324666170404150351.

9. Ahmad, A.; Raish, M.; Alkharfy, K.M.; Alsarra, I.A.; Khan, A.; Ahad, A.; Jan, B.L.; Shakeel, F. Solubility, solubility parameters and solution thermodynamics of thymoquinone in different mono solvents. Journal of Molecular Liquids 2018, 272, 912-918, https://doi.org/10.1016/j.molliq.2018.10.104.

10. Darakhshan, S.; Bidmeshki Pour, A.; Hosseinzadeh Colagar, A.; Sisakhtnezhad, S. Thymoquinone and its therapeutic potentials. Pharmacological Research 2015, 95-96, 138-158, https://doi.org/10.1016/j.phrs.2015.03.011.

11. Heiss, A.G.; Oeggl, K. The oldest evidence of Nigella damascena L. (Ranunculaceae) and its possible introduction to central Europe. Vegetation History and Archaeobotany 2005, 14, 562570, https://doi.org/10.1007/s00334-005-0060-4.

12. Khader, M.; Eckl, P.M. Thymoquinone: an emerging natural drug with a wide range of medical applications. Iran J Basic Med Sci 2014, 17, 950-957.

13. Ahmad, A.; Husain, A.; Mujeeb, M.; Khan, S.A.; Najmi, A.K.; Siddique, N.A.; Damanhouri, Z.A.; Anwar, F. A review on therapeutic potential of Nigella sativa: A miracle herb. Asian Pacific Journal of Tropical Biomedicine 2013, 3, 337-352, https://doi.org/10.1016/S2221-1691(13)60075-1.

14. El-Dakhakhny, M. Studies on the chemical constitution of Egyptian Nigella sativa L. seeds. II $^{1}$ ) the essential oil. Plant Med1963, 11, 465-470, https://doi.org/10.1055/s-0028-1100266.

15. Hassanien, M.F.R.; Assiri, A.M.A.; Alzohairy, A.M.; Oraby, H.F. Health-promoting value and food applications of black cumin essential oil: an overview. Journal of Food Science and Technology 2015, 52, 6136-6142, https://doi.org/10.1007/s13197-015-1785-4.

16. Banerjee, S.; Padhye, S.; Azmi, A.; Wang, Z.; Philip, P.A.; Kucuk, O.; Sarkar, F.H.; Mohammad, R.M. Review on Molecular and Therapeutic Potential of Thymoquinone in Cancer. Nutrition and Cancer 2010, 62, 938-946, https://doi.org/10.1080/01635581.2010.509832.

17. Goyal, S.N.; Prajapati, C.P.; Gore, P.R.; Patil, C.R.; Mahajan, U.B.; Sharma, C.; Talla, S.P.; Ojha, S.K. Therapeutic Potential and Pharmaceutical Development of Thymoquinone: A Multitargeted Molecule of Natural Origin. 2017, 8, 1-19, https://doi.org/10.3389/fphar.2017.00656.

18. Alsamarai, A.M.; Abdulsatar, M.; Ahmed Alobaidi, A.H. .Evaluation of topical black seed oil in the treatment of allergic rhinitis. Antiinflamm Antiallergy Agents Med Chem 2014, 13, 75-82, https://doi.org/10.2174/18715230113129990014. 
19. Torres, J.; Parthasarathy, K.; Lin, X; Saravanan, R ; Kukol, A. Model of a putative pore: the pentameric ahelical bundle of SARS coronavirus E protein in lipid bilayers. Biophys J 2006, 91, 938-947, https://doi.org/10.1529/biophysj.105.080119.

20. DeDiego, M.L.; Alvarez, E.; Almazan, F.; Rejas, M.T.; Lamirande, E. A severe acute respiratory syndrome coronavirus that lacks the $\mathrm{E}$ gene is attenuated in vitro and in vivo. J Virol 2007, 81, 1701-1713, https://doi.org/10.1128/JVI.01467-06.

21. Tahir ul Qamar, M.; Alqahtani, S.M.; Alamri, M.A.; Chen, L.-L. Structural basis of SARS-CoV-2 3CLpro and anti-COVID-19 drug discovery from medicinal plants. Journal of Pharmaceutical Analysis 2020, https://doi.org/10.1016/j.jpha.2020.03.009.

22. Pervushin, K.; Tan, E.; Parthasarathy, K.; Lin, X.; Jiang, F.L.; Yu, D.; Vararattanavech, A.; A.T.W, Soong.; Liu, D.X.; Torres, J. Structure and inhibition of the SARS coronavirus envelope protein ion channel. PLoS Pathog 2009, 5, https://doi.org/10.1371/journal.ppat.1000511.

23. Dediego, M.L.; Pewe, L; Alvarez, E.; Rejas, M.T.; Perlman, S. Pathogenicity of severe acute respiratory coronavirus deletion mutants in hACE- 2 transgenic mice. Virology 2008, 376, 379-389. https://doi.org/10.1016/j.virol.2008.03.005.

24. Curtis, K.M.; Yount, B.; Baric, R.S. Heterologous Gene Expression from Transmissible Gastroenteritis Virus Replicon Particles. J Virol 2002, 76, 1422-1434,https://doi.org/10.1128/jvi.76.3.1422-1434.2002.

25. Ortego, J.; Escors, D.; Laude, H.; Enjuanes L. Generation of a replication competent; propagation-deficient virus vector based on the transmissible gastroenteritis coronavirus genome. J Virol 2002, 76, 11518-115, https://doi.org/10.1128/jvi.76.22.11518-11529.2002.

26. Wu, F.; Zhao, S.; Yu, B.A new coronavirus associated with human respiratory disease in China. Nature 2020, 579, 265-269,https://doi.org/10.1038/s41586-020-2008-3.

27. Dimitrov, I.; Bangov, I.; Flower, D.R.; Doytchinova, I. AllerTOP v.2. A server for in silico prediction of allergens. J Mol Model 2014, 20, https://doi.org/10.1007/s00894-014-2278-5.

28. Geourjon, C.; Deléage, G. SOPMA: significant improvements in protein secondary structure prediction by consensus prediction from multiple alignments. Bioinformatics 1995, 11, 681-684, https://doi.org/10.1093/bioinformatics/11.6.681.

29. Shin, W.H.; Lee, G.R.; Heo, L.; Lee, H.; Seok, C.Prediction of Protein Structure and Interaction by GALAXY protein modeling programs. Bio Design 2014, 2, 1-11.

30. Pontius, J.; Richelle, J.; Wodak, S.J. Deviations from Standard Atomic Volumes as a Quality Measure for Protein Crystal Structures. Journal of Molecular Biology 1996, 264, 121-136, https://doi.org/10.1006/jmbi.1996.0628.

31. Wiederstein, M.; Sippl, M.J.ProSA-web: interactive web service for the recognition of errors in threedimensional structures of proteins. Nucleic Acids Res 2007, 35, W407-W410, https://doi.org/10.1093/nar/gkm290.

32. Pugalenthi, G.; Archunan, G.; Sowdhamini, R. DIAL: a web-based server for the automatic identification of structural domains in proteins. Nucleic Acids Res 2005, 33, W130-W132, https://doi.org/10.1093/nar/gki427.

33. Yin, Z.;Song, Y.;Rehse, P.H. Human Plk1-PBD in complex with Thymoquinone at the phophopeptide binding site. WPDB 2012 , https://doi.org/10.2210/pdb4HCO/pdb.

34. Thompson, M.A.Molecular docking using Argus Lab; an efficient shape-based search algorithm and the AScore scoring function. In: Proceedings of the ACS meeting. Philadelphia 172; CINF 42; PA. 2004.

35. Schneidman-Duhovny, D.; Inbar, Y.; Nussinov, R.; Wolfson, H.J. PatchDock and SymmDock: servers for rigid and symmetric docking. Nucleic Acids Res 2005, 33, W363-W367,https://doi.org/10.1093/nar/gki481.

36. Reji, M.I. Computation of Molecular Weight of Proteins. Proceedings of UGC Sponsored Third National Conference on Modern Trends in Electronic Communication \& Signal Processing (NCMES-2013). Published By: Department of Electronics, BPC College, Piravom, Kochin, Keralam, India.

37. Li, L.; Vorobyov, I.; Allen, T.W. The different interactions of lysine and arginine side chains with lipid membranes. The journal of physical chemistry B 2013, 117, 11906-11920, https://doi.org/10.1021/jp405418y.

38. Gasteiger, E.; Hoogland, C.; Gattiker , A.; Duvaud, S.; Wilkins, M.R.; Appel, R.D.; Bairoch , A. Protein identification and analysis tools on the ExPASy server In: The Proteomics Protocols Handbook. Walker, J.M. editor Clifton, U.K: Humana Press 2005; pp. 571-607.

39. Neote, K; DiGregorio, D.; Mak, J.Y.; Horuk, R.; Schall, T.J..Molecular cloning; functional expression; and signaling characteristics of a C-C chemokine receptor. Cell 1993, 72, 415-425 ,https://doi.org/10.1016/00928674(93)90118-a.

40. Gao, J.L.; Kuhns, D.B.; Tiffany, H.L. Structure and functional expression of the human macrophage inflammatory protein 1 alpha/RANTES receptor. $J \quad$ Exp Med 1993, 177, 14211427,https://doi.org/10.1084/jem.177.5.1421.

41. Torres, J.;Surya, W.;Li, Y. NMR structure of the SARS Coronavirus E protein pentameric ion channel. WPDB 2017, https://doi.org/10.2210/pdb5X29/pdb.

42. Branden, C.; Tooze, J. Introduction to Protein Structure. Garland Publishing; New York 302; Biochem Edu 1991; pp. 121-122. 
43. Al-Karadaghi, S.Protein Secondary Structure: $\alpha$-Helices and $\beta$-Sheets. Depatment of Biochemistry and Structural Biology; Centre for Molecular Protein Science, Lund University, Sweden. Accessed on 28 May 2020. https://proteinstructures.com/Structure/Structure/secondary-sructure.html

44. Iacovache, I.; Bischofberger, M.; van der Goot, F.G. Structure and assembly of pore-forming proteins. Current Opinion in Structural Biology 2010, 20, 241-246, https://doi.org/10.1016/j.sbi.2010.01.013.

45. Katia, C.; Uris, R.; Ana, J.G-S.Assembling the puzzle: Oligomerization of $\alpha$-pore forming proteins in membranes. Biochimica et Biophysica Acta (BBA)-Biomembranes 2016, 1858,457466,https://doi.org/10.1016/j.bbamem.2015.09.013.

46. Verdiá-Báguena, C.; Nieto-Torres, J.L.; Alcaraz, A.; DeDiego, M.L.; Torres, J.; Aguilella, V.M.; Enjuanes, L. Coronavirus E protein forms ion channels with functionally and structurally-involved membrane lipids. Virology 2012, 432, 485-494,https://doi.org/10.1016/j.virol.2012.07.005.

47. Laventie, B.J.; Potrich, C.; Atmanène, C.; Saleh, M.; Joubert, O.; Viero, G.; Bachmeyer, C.; Antonini, V.; Mancini, I.; Cianferani-Sanglier, S.; Keller, D.; Colin, Didier A.; Bourcier, T.; Anderluh, G.; van Dorsselaer, A.; Dalla Serra, M.; Prévost, G. p-Sulfonato-calix[n]arenes inhibit staphylococcal bicomponent leukotoxins by supramolecular interactions. Biochemical Journal 2013, 450, 559-571, https://doi.org/10.1042/BJ20121628.

48. LaRosa, S.P.; Opal, S.M. Sepsis Strategies in Development. Clinics in Chest Medicine 2008, 29, 735-747, https://doi.org/10.1016/j.ccm.2008.06.007.

49. Torres, J.; Maheswari, U.; Parthasarathy, K.; Ng, L.; Liu, D.X.; Gong, X. Conductance and amantadine binding of a pore formed by a lysine-flanked transmembrane domain of SARS coronavirus envelope protein. Protein Science 2007, 16, 2065-2071,https://doi.org/10.1110/ps.062730007.

50. Nieto-Torres, J.L; Verdiá-Báguena, C.; Jimenez-Guardeño, J.M.; Regla-Nava, J.A.; Castaño-Rodriguez, C.; Fernandez-Delgado, R. Severe acute respiratory syndrome coronavirus E protein transports calcium ions and activates the NLRP3 inflammasome. Virology 2015, 485, 330-9, https://doi.org/10.1016/j.virol.2015.08.010.

51. Roberts, K.L.; Leser, G.P.; Ma, C.; Lamb, R.A. The amphipathic helix of influenza a virus M2 protein is required for filamentous bud formation and scission of filamentous and spherical particles. $J$ Virol 2013, 87, 9973-82, https://doi.org/10.1128/JVI.01363-13.

52. Berg, J.M.; Tymoczko, J.L.; Stryer, L.Biochemistry. 7th revised International Edition;W.H. Freeman \& Company 2012.

53. Rosenthal, J.; Daniel, G.N. Why Does Combining Hydrogen and Oxygen Typically Produce Water Rather than Hydrogen Peroxide. Scientific American (2006). Accessed on 28 May 2020. https://www.scientificamerican.com/article/why-does-combining-hydrog/.

54. Klaholz, B.; Moras D. C---HO hydrogen bonds in the nuclear receptor RAR gamma -a potential tool for drug selectivity. Structure2002, 10, 1197-1204,https://doi.org/10.1016/s0969-2126(02)00828-6.

55. Alkorta, I.; Blanco, F.; Elguero, J.; Dobado, J.A.; Ferrer, S.M.; Vidal, I. Carbon ‥Carbon Weak Interactions. The Journal of Physical Chemistry A 2009, 113, 8387-8393,https://doi.org/10.1021/jp903016e.

56. [Martinovich, G.G.; Martinovich, I.V.; Vcherashniaya, A.V.; Shadyro, O.I.; Cherenkevich, S.N. Thymoquinone, a biologically active component of Nigella sativa, induces mitochondrial production of reactive oxygen species and programmed death of tumor cells. Biophysics 2016, 61, 963-970, https://doi.org/10.1134/S0006350916060154.

57. National Cancer Institute. Reactive Oxygen Species. Bethesda MD. Accessed on 28 May 2020. https://www.cancer.gov/publications/dictionaries/cancer-terms/def/reactive-oxygen-species.

58. Barkat, M.A.; Harshita.; Ahmad , J.; Khan, M.A.; Beg, S.; Ahmad, F.J. Insights into the Targeting Potential of Thymoquinone for Therapeutic Intervention Against Triple-negative Breast Cancer. Curr Drug Targets 2018, 19, 70-80,https://doi.org/10.2174/1389450118666170612095959.

59. Hosny, N.A.; Sherif, Y. Molecular Docking Study on Some Isonicotinoyl Hydrazide Derivatives as Potential Inhibitors of COVID-19. Letters in Applied NanoBioscience 2020, 9, 1217- 1224, https://doi.org/10.33263/LIANBS93.12171224. 